\title{
Velocity measurements using UDV in rotating annulus subjected to quasi 2D temperature variations
}

\author{
Sunil Yadav ${ }^{1 *}$, Ayan Banerjee ${ }^{2}$,Tapobrata Dey ${ }^{1}$ and Sridhar Balasubramanian ${ }^{2}$ \\ ${ }^{1}$ Department of mechanical engineering D.Y Patil College of Engineering, Akurdi India 411044 \\ ${ }^{2}$ Department of Mechanical Engineering, Indian Institute of Technology Bombay, Mumbai,India 400076 \\ Accepted 15 June 2016, Available online 20 June 2016, Special Issue-5 (June 2016)
}

\begin{abstract}
An experimental study was carried on in a rotating cylindrical annulus having temperature variation along both radial and vertical directions. The experiment set up comprises with an inner cylinder which is kept at cold temperature, outer cylinder and a bottom plate where heating is provided along periphery. This inner cooling and peripheral heating represent poles and equator respectively. This enables study on baroclinic instability in laboratory to have better understanding of effect of rotation on heat transport and turbulent convection in atmosphere. In this experiment, UDV (Ultrasonic Doppler Velocimeter) which works on Doppler principle was used to find velocity along the centre axis of cylinder ( $Z$ axis) and azimuthal velocity ( $Y$ axis). This study was carried out by providing different angular speed i.e. 8.46rpm, 12.9rpm and $17.34 \mathrm{rpm}$ by using a VFD (variable frequency device) to table on which concentric cylinder are placed. By using 2D UDV, velocities at different vertical points were captured. By conducting this experiment it was found that fluctuation in velocity increases in $z$ axis where as it decrease in Y axis. Deapth from top surface velocity in $Z$ axis show less irregularity as compared to velocity along $Y$ axis.
\end{abstract}

Keywords: Rotation, UDV, baroclinic, instability, doppler effect, Coriolis effect.

\section{Introduction}

Thermal convection plays an important role in transfer of heat from equator (which is at high temperature) to pole (which is at low temperature). Turbulent thermal convection in the presence of background rotation is an important fluid dynamic problem which has got wide attention in recent decades. Heat transport in rotating fluid with radial temperature gradient leads to baroclinic instability which is the main mechanism of heat transport in planetary atmosphere. Main objective of this study is to observe the effect of Coriolis and temperature gradient on the velocity of annular rotating flow. Chandrasekhar et al, have studied the effect of Coriolis force on heat transport mechanism (S. Chandrasekhar,1953). Effect of fluid velocity and rotation on close assembly which is provided with thermal temperature gradient falls under Rayleigh benard convection (RBC) (S. Chandrasekhar,1953). Later on Rossby observed the effect of Taylor number, Rayleigh number, Prandtl number and Rossby number on fluid stability and thermal convection (H.T Rossby,1969) and qualitative flow vortices visualization of RBC is done by fang et al (Fang Zhong et al 1993).

When a fluid in a closed annuals space is subjected to temperature gradient in which heating is provided

*Corresponding author: Sunil Yadav from outside and cooled from inside then turbulent cascade is observed along with baroclinic instability ( $R$. D. Wordsworth et al 2008). In a closed annular cylinder having a temperature gradient along the radius shows an alternate convergent and divergent zone (Uwe Harlander et al,2011). Lecher performed a experiment on free stream of annular rotating cylinder which is cold from inside and heated from outer side and he observed transition from axisymmetric to regular wave regimes by using thermal image processing (Th. von et al,2005).

This study deals with capturing of velocity at different height by using ultrasonic Doppler velocimeter(UDV). It further shows the effect of angular speed on velocity pattern. Experiment is conducted on 8.46rpm, 12.9rpm and $17.34 \mathrm{rpm}$ by using Variable frequency device (VFD).

\section{Experiment Setup}

Experimental setup consist of a rotating table on which concentric cylinder is placed. Rotating motion to the table is provide by using electric motor in which speed is controlled by using variable frequency device (VFD). Outer cylinder which is made up of acrylic is provided with circumferential heating at bottom by using a heat coil at bottom plate. Two rotor-stator connectors exist above the upper plate and beneath the lower plate. The 
rotor-stator connector above the upper plate connects the rotating frame with electricity from the stationary frame. Inner cylinder filled up with water and chiller is installed for cold water circulation so that the water within cylinder remains cold all the time. The rotorstator connector below the lower plate helps for water circulation between rotating turn table and chiller in stationary frame.

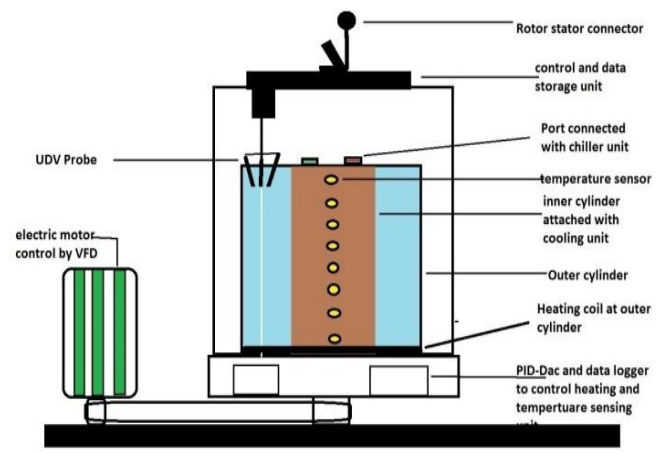

Fig.1 schematic diagram of experimental setup

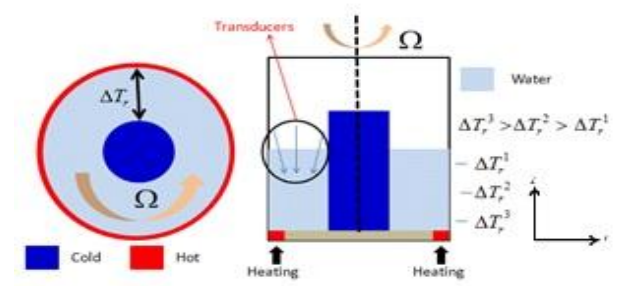

Fig.2 schematic representation of working setup

Both cylinders are placed above the heating pad. A Variac is used for proper working of heating pad. Water is used as working fluid $(\mathrm{Pr}=7)$. Height and diameter of outer cylinder are $31 \mathrm{~cm}$ and $40 \mathrm{~cm}$ respectively. Diameter of inner cylinder is $10 \mathrm{~cm}$ whereas thickness of aluminum sheet is $5 \mathrm{~mm}$.

Doppler ultrasound velocimetry or Ultrasonic Doppler veloctimeter (UDV) is used to find particle velocity. UDV works on principle of Doppler effect in which speed of particle is estimated by calculating Doppler frequency.

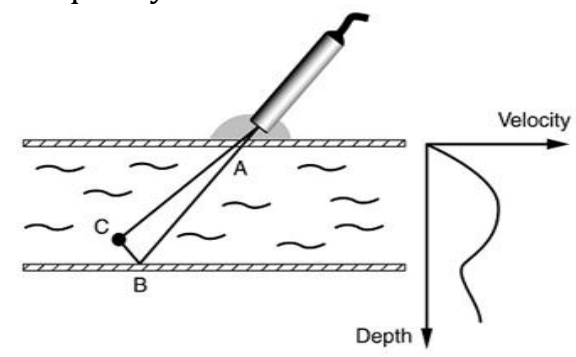

Fig .3 Working principle of UDV

2D UDV is used to plot velocity along the axis of cylinder called $\mathrm{Z}$ velocity and velocity along azimuthal plane called Y velocity. UDV consist of one emitting probe and two receiving probe. All the three probes are held at $20^{\circ}$ from vertical axis and angle between receiving probe is $40^{\circ}$.

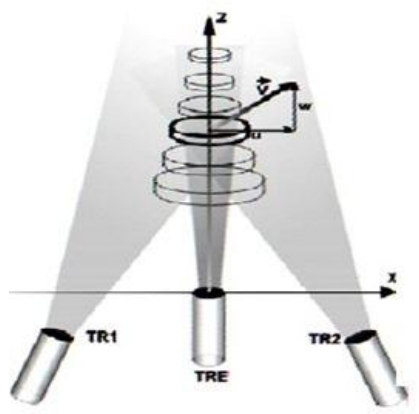

Fig 4. UDV probe arrangement for 2D velocity measurement

UDV probes and parameters were set as per table1 specification which is obtained by simulation as per need. Then different rotation speed at 1 VFD (8.46rpm) 1.5 VFD (12.9rpm) and 2 VFD (17.34rpm) is provided to the assembly and at different elevation velocity data is captured by UDV. Here for analysis purpose velocity of only $5 \mathrm{~mm}, 50 \mathrm{~mm}$ and $105 \mathrm{~mm}$ from the top is consider.

Table.1 Ultrasonic Doppler velocity-meter controlling Parameters

\begin{tabular}{|c|c|}
\hline Parameters & Specification \\
\hline Emitter Probe specification & TR0408LS, 4MHz, 8mm \\
\hline Pulse Repetition Frequency & $3000 \mu \mathrm{s}$ \\
\hline Doppler angle & $20^{\circ}$ \\
\hline $\begin{array}{c}\text { Angle between emitter and } \\
\text { receiver probe }\end{array}$ & $20^{0}$ \\
\hline $\begin{array}{c}\text { Distance between emitter } \\
\text { and receiver probe }\end{array}$ & $38 \mathrm{~mm}$ \\
\hline Depth to measure & $5 \mathrm{~mm}$ to $110 \mathrm{~mm}$ from top \\
\hline
\end{tabular}

\section{Result and discussion}

After achieving desired baroclinic phenomenon UDV is switch on to capture the velocity for 64000 velocity time instant profile. Both vertical i.e along the axis and azimuthal velocity is captured.

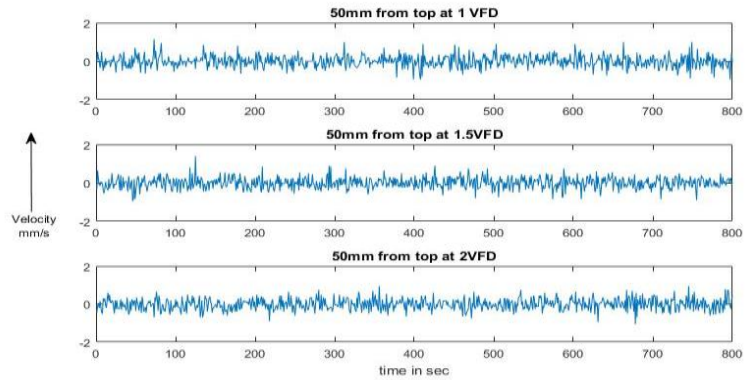

Fig 5. Z velocity (along the centre axis) plot at $50 \mathrm{~mm}$ from top (on $\mathrm{x}$ axis time is plotted in sec and $\mathrm{Z}$ axis velocity magnitude is plotted in $\mathrm{mm} / \mathrm{s}$ ) 


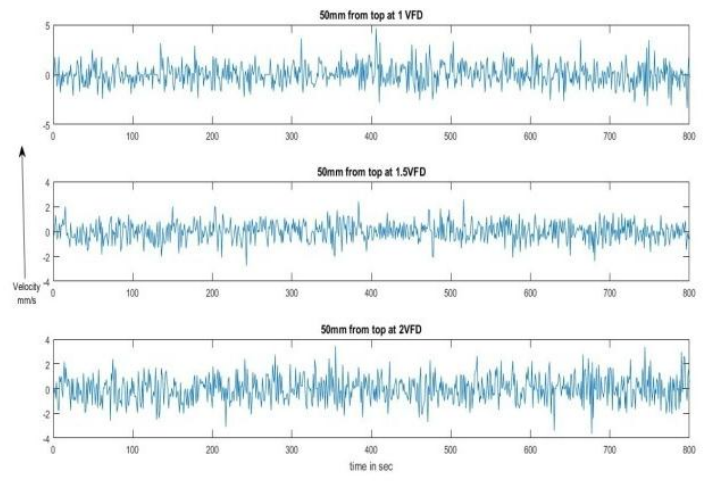

Fig 6. Y velocity (Perpendicular to centre axis) plot at $50 \mathrm{~mm}$ from top (on $\mathrm{x}$ axis time is plotted in sec and $\mathrm{Y}$ axis velocity magnitude is plotted in $\mathrm{mm} / \mathrm{s}$ )

As there is formation of alternate convergent and divergent zone which causes high fluctuation in velocity. Usually $2 / 7$ and $5 / 7$ correlation is used to express the relation between Nusselt number Rayleigh and Taylor number.

The main governing non-dimensional numbers for this problem are: Thermal Rossby number $\left(\mathrm{R}_{\mathrm{OT}}\right)$ which is the ratio of buoyant force to Coriolis force, and the Taylor number (Ta) which is the ratio of the Coriolis force to viscous forces, and characterizes the earth's rotation. The rotation inhibits the onset of convective turbulence as turbulence in such rotating flows is twodimensional. The flow regime is classified as: (a) Axisymmetric flow, (b) Non-axisymmetric flow (i.e. Regular baroclinic wave and Irregular baroclinic wave) (Fig. 8). Different numbers of lobes are also formed depending on the values of Ta and $R_{0 T}$. The $R_{0 T}-T a$ regime diagram for fluid like water (Fig. 7) which shows the well investigated anvil shape of the 'transition curve'. The 'transition curve' indicates the transition from azimuthally symmetric (i.e. axisymmetric, no waves present) to non-axisymmetric wavy regime, finally to irregular, chaotic flow (geostrophic turbulence).

$T a=\frac{4 \Omega^{2}(b-a)^{5}}{v^{2} d} \quad R o_{T}=\frac{g \alpha d \Delta T}{\Omega^{2}(b-a)^{2}}$
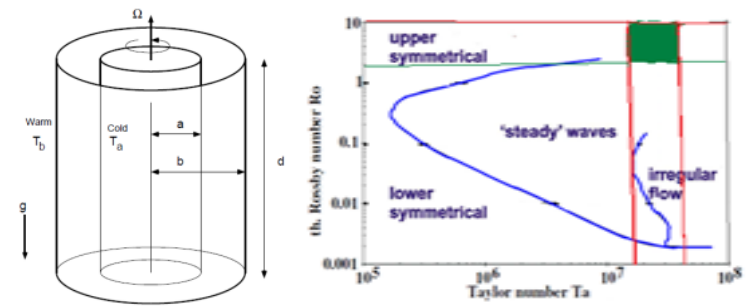

Fig 7: Schematic diagram of rotating baroclinic annulus experimental set up. (Lewis, Nagata, 2011); $\mathrm{R}_{0 \mathrm{~T}}-\mathrm{Ta}$

regime diagram of water in differentially heated rotating annulus experiment for flat bottom end walls (Fowlis and Hide, 1965, modified by Larcher, Egbers, 2005).The 'green box' denotes the range of Ta and $\mathrm{R}_{0 \mathrm{~T}}$ in our experiments $\left(\Delta \mathrm{T}=5-15^{\circ} \mathrm{C}\right.$ and $\Omega=4.9-8.46 \mathrm{rpm}$ respectively)
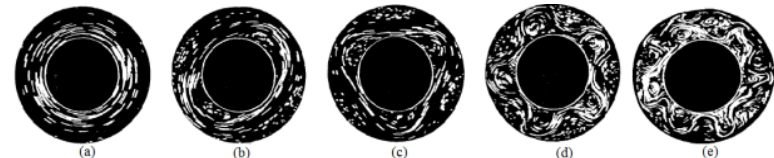

Fig 8: Flow behavior in different regimes:

(a) axisymmetric flow (b,c) non-symmetric flow of wave number two and three (d) transition flow (e) irregular flow (geostrophic turbulence) (Hide, Mason,

1975)

Natural convection leads to vertical movement of water from bottom to top as system is subjected to temperature difference between top and bottom surface. Coriolis force which are developed due to rotation of system leads to formation of alternate high and low pressure zone as shown in figure 9.
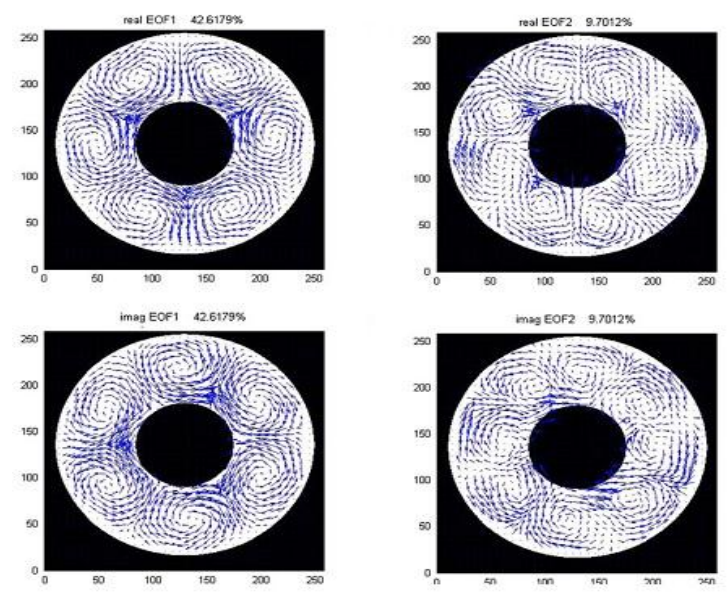

Fig 9. Formation of alternate high pressure and low pressure zone in rotating annular flow

\section{Conclusions}

Due to temperature difference between top and bottom periphery natural convection occurs which leads to movement of hot water from bottom to top which is similar to Rayleigh Benard convection (RBC). As frame is rotating, Coriolis force play significant role which leads to formation of different cyclonic lobes of high and lower pressure zone. Here Due to presence of both RBC and Coriolis force Variation in magnitude of $\mathrm{z}$ velocity is very less as compared to y velocity along y axis and also as angular speed increases $\mathrm{z}$ velocity decrease in magnitude.

\section{Acknowledgements}

Authors thank to Geophysical and Multiphase Flow Lab (GMFL) at Indian Institute of Technology, Bombay for sponsoring this project.

\section{References}

S. Chandrasekhar(1953), The instability of a layer of fluid heated below and subject to Coriolis forces, Proc. R. Soc. Lond. A, 217, pp. 306-327. 
H.T Rossby(1969), A study of Benard convection with and without rotation Journal of Fluid Mechanics ,36(2),pp. 309-335

Fang Zhong, Robert Ecke, and Victor Steinberg (1993),Asymmetric Modes and the Transition to Vortex Structures in Rotating Rayleigh-Benard Convection, Journal of fluid mechanics , 67, pp. 135-149.

R. D. Wordsworth, P. L. Read, and Y. H. Yamazaki.(2008) Turbulence, waves, and jets in a differentially heated rotating annulus experiment, Physics of fluids, 20, pp 126602.

Uwe Harlander Thomas von Larcher(2011), PIV- and LDVmeasurements of baroclinic wave interactions in a thermally driven rotating annulus,Exp Fluids, 51,pp.37-49.

Th. von Larcher and C. Egbers(2005), Experiments on transitions of baroclinic waves in a differentially heated rotating annulus, Nonlinear Processes in WSGeophysics ,12, pp. 103-117.
T. H. Solomon Jerry P. Gollub (1991), Thermal boundary layers and heat flux in turbulent convection: the role of recirculating flows,Haverford College Haverford Scholarship faculty publication, 43, pp 12-25.

Moses L. Ng and J.P. Hartnett (1986), Natural convection in power-law fluids, int. comm. Heat mass transfer,13,pp 115120.

Richard J. A. M. Grossmann et n (2013), The unifying theory of scaling in thermal convection: The updated prefactors, Physics of fluid dynamics, 2,pp

Sridhar Balasubramanian and Robert E. Ecke (2013), Experimental Study of Rayleigh-Bé nard Convection in the Presence of Rotation, International Journal of Materials, Mechanics and Manufacturing, 1, No. 2.

Yuanming Liu and Robert E. Ecke (2009), Heat transport measurements in turbulent rotating Rayleigh-Bénard convection, Physical review e 80, 036314.

Marke. Bastin and peterl. Read (1997), A laboratory study of baroclinic waves and turbulence in an internally heated rotating fluid annulus with sloping end walls, Journal of fluid mechanics, 339, pp 173-198. 